\title{
Beta beams
}

\author{
ELENA WILDNER ${ }^{* \dagger}$ \\ CERN \\ E-mail: elena.wildnerdcern.ch
}

The recent discovery of neutrino oscillations, has implications for the Standard Model of particle physics. Knowing the contribution of neutrinos to the Standard Model, needs precise measurements of the parameters governing the neutrino oscillations. The EUROnu Design Study (European Commision FP7 program) will review three facilities (the so-called Super-Beams, Beta Beams and Neutrino Factories) and perform a cost assessment that, coupled with the physics performance, will give means to the European research authorities to make a decision on future European neutrino oscillation facility. "Beta Beams" produce collimated pure electron (anti)neutrino by accelerating beta active ions to high energies and having them decay in a storage ring. EUROnu Beta Beams are based on CERN's infrastructure and existing machines. Using existing machines is an advantage for the cost evaluation, however, this choice is also constraining the Beta Beams. After a brief introduction to beta beams, recent work to make the Beta Beam facility a solid option will be mentioned: tuning of beta beam parameters to give high fluxes and to enhance the physics reach (production of Beta Beam isotopes, the $60 \mathrm{GHz}$ pulsed ECR source development, the integration into the LHC-upgrades, ensure the high intensity ion beam stability).

The 2011 Europhysics Conference on High Energy Physics-HEP 2011,

July 21-27, 2011

Grenoble, Rhône-Alpes France

\footnotetext{
* Speaker.

${ }^{\dagger}$ Work done within the European Commission Frame-work Programme 7 Design Study: EUROnu, Project Number 212372 .
} 


\section{Introduction}

Production of pure and intense (anti-)neutrino beams from beta decay of radioactive isotopes circulating at high energy in a race track shaped storage ring (the Decay Ring, DR) was proposed in 2002 [1]. The neutrino energy depends on the reaction Q-value and of the chosen relativistic $\gamma$ boost of the stored isotopes. Acceleration of ions is commonly done in accelerators. The isotope pair ${ }^{6} \mathrm{He} /{ }^{18} \mathrm{Ne}$ (Q-values of $3.5 \mathrm{MeV}$ and $3.3 \mathrm{MeV}$ ) has been selected for Beta Beam studies in the CERN Complex, see Fig. 1.

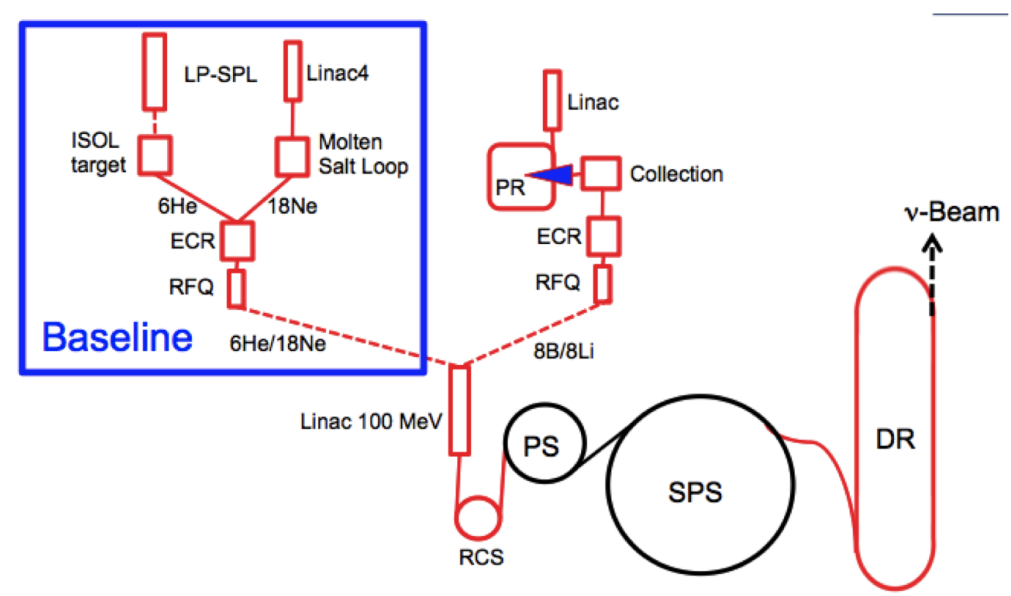

Figure 1: Layout of the CERN Beta Beam, where the PS and the SPS are existing machines. The baseline scenario is to use ${ }^{6} \mathrm{He} /{ }^{18} \mathrm{Ne}$ (low-Q) with neutrinos to Fréjus. ${ }^{8} \mathrm{Li} /{ }^{8} \mathrm{~B}$ (high-Q) would need a detector to Gran Sasso or Canfranc. The magnetic field of the main magnet in the Decay Ring is $6 \mathrm{~T}$, the circumference is $6900 \mathrm{~m}$, the straight section length $2500 \mathrm{~m}$, and the $\gamma$-boost is 100 for all ions.

The CERN SPS allows a maximum $\gamma$-value of $150\left({ }^{6} \mathrm{He}\right)$ or $250\left({ }^{18} \mathrm{Ne}\right)$. However, the choice of energy, corresponding to a $\gamma$-value of 100 , was made to optimize the physics reach at a baseline $130 \mathrm{~km}$ from CERN where there is a possibility to locate a Mton water Cherenkov detector, MEMPHYS, for astrophysics (LAGUNA study [2], in the Fréjus tunnel). The choice of a detector (location and technology) for astrophysics is crucial for the medium and long baseline neutrino oscillation experiments: without this synergy, price and efforts for the accelerator beam based neutrino oscillation physics facility would be considerably increased. Using ${ }^{6} \mathrm{He} /{ }^{18} \mathrm{Ne}$ and a detector at some $130 \mathrm{~km}$ we would have very good physics reach for $\mathrm{CP}$ violation since no matter effects are present. Production of another isotope pair ${ }^{8} \mathrm{Li} /{ }^{8} \mathrm{~B}$ for $\bar{v}_{e} / v_{e}$ with Q-values of $13.0 \mathrm{MeV}$ and 13.9 MeV [3] is also studied, which suggests that the distance to the detector for these isotopes would be around $700 \mathrm{~km}$ (CERN-Canfranc or CERN-LNGS). However with present technologies, production rates of these isotopes are not yet compatible with needs for good physics reach.

For energies of the neutrinos generated by Beta Beams (around $350 \mathrm{MeV}$ ) the atmospheric background is large. To suppress this background the neutrino beam pulses from the DR have to be very short. This is a technical constraint for the DR. However, calculations using today's experimental hints on the value of $\sin ^{2} 2 \theta_{13}$ [4] tell us that we may relax the duty factor of the DR and consequently increase the flux of neutrinos in the detector. Furthermore, optimization of $\gamma$-boosts and preferred Q-values may open new possibilities for physics. 


\section{Developments for high physics performance}

The CERN Beta Beam facility is based on CERN infrastructure and machines and on existing technologies which is of interest to keep the cost of the neutrino facility low. Research and experiments are now addressing the optimization of isotope production and the consolidation of an overall acceleration scenario in view of a performance/cost analysis (EUROv, European Framework Programme FP7 [5] and [6]).

Results from experiments show that ${ }^{6} \mathrm{He}$ can be produced in sufficient rates with an available beam power of $200 \mathrm{~kW}$ (SPL, $2 \mathrm{GeV}$ ) [7]. Production of ${ }^{18} \mathrm{Ne}$ is presently (fall 2011) measured at ISOLDE $^{1}$ using a molten NaF salt loop [8].

For large $\sin ^{2} 2 \theta_{13}$ [4], systematic uncertainties and neutrino flux are important aspects for optimization of the neutrino facilities. Since the atmospheric suppression is now less important, the number of bunches in the Beta Beam DR may be increased with less intensity per bunch (for the same circulating beam intensity). Lower bunch intensities favor beam stability. Calculations for optimization of suppression factors of the neutrino beam (the ratio of beam/no beam in the detector) for a given possible flux have been performed to get the possible relaxation of the duty factor of the Decay Ring ion beam structure. The dependence of the flux on physics has also been looked at. This is shown in Fig. 2. From these calculations we presently study a suppression factor of $2 \%(0.05 \%$ was the originally suggested duty factor).

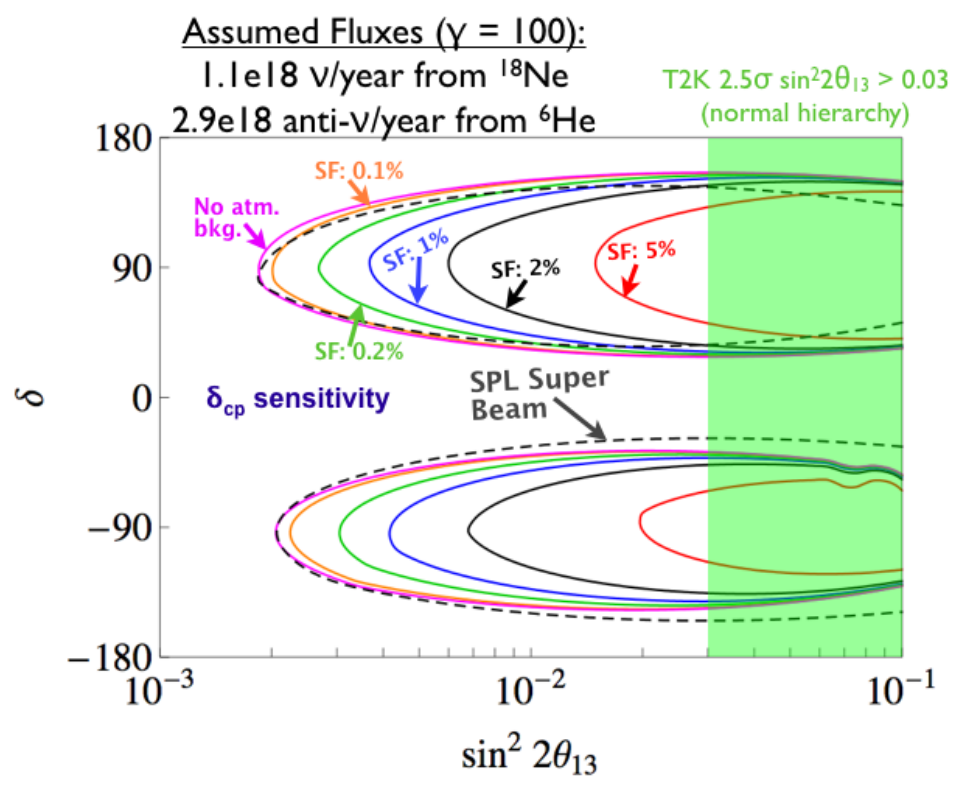

Figure 2: Estimation of the atmospheric suppression factors for the Beta Beam. If we assume that $\sin ^{2} 2 \theta_{13}$ is large we can tune the Beta Beam parameters for a duty factor of $2 \%$ without considerable impact on the physics performance.

To explore new possibilities for the Beta Beam with the relaxed conditions in the Decay Ring for large $\sin ^{2} 2 \theta_{13}$, the cycling and the beam bunching in the accelerators are studied. Space charge consideration for the Beta Beam has led to a design energy of $3.5 \mathrm{GeV}$ for the injection into the

\footnotetext{
${ }^{1}$ Isotope Separator On Line-DEtector
} 
CERN PS, however the upgrade of the CERN injectors for LHC will bring the injection energy of the PS to only $2.0 \mathrm{GeV}$. Measurements of tune shifts and of emittance growth, as a function of the intensity, are ongoing [9] as well as optimization of the bunch structure in the SPS (beam stability) [10]. The Decay Ring has been optimized to ensure beam stability [11].

Studies for radio protection of the RCS, the PS and the Decay Ring have been carried out [12]. No show-stopper has been found, neither for radio-protection nor equipment. The PS upgrade studies will permit radiological studies also for the Beta Beam. The intensity of the Beta Beam is optimized by end to end simulations.

\section{Conclusion}

The CERN Beta Beam is a unique option for precision measurements of neutrino oscillation parameters, in particular measurements of $\mathrm{CP}$ violation in the leptonic sector. The main research issues, ion production, flux to detectors and end-to-end simulations of the ion beam acceleration through the Beta Beam Complex are all addressed. Experiments to verify production of ${ }^{6} \mathrm{He}$ has shown that we can reach wanted production rates, production of ${ }^{18} \mathrm{Ne}$ is presently being measured. Stable beams in the machines will be provided by optimization of beam parameters and measurements.

\section{Acknowledgments}

We acknowledge the financial support of the European Community under the European Commission Framework Program 7 Design Study: EUROnu, Project Number 212372. The EC is not liable for any use that may be made of the information herein.

\section{References}

[1] P. Zucchelli, Phys. Lett. B532 (2002) 166-172.

[2] LAGUNA, Large Apparatus studying Grand Unification and Neutrino Astrophysics, FP7 INFRA-2007-2.1-01, ref.212343.

[3] C.Rubbia et al. Nucl. Instrum. Meth. A568 (2006) 475-487.

[4] K.Abe et al., arXiv:1106.2822.

[5] EUROnu-A High Intensity Neutrino Oscillation Facility in Europe, FP7-INFRASTRUCTURES-2007-1, ref. 212372.

[6] E. Wildner et al., Conf. Proc. IPAC'11, WEPS024.

[7] T.Y.Hirsh et al., PoSNUFACT08(2008) 090.

[8] T.Stora, to be published, AIP Conf. Proc. NUFACT11.

[9] S.Gilardoni et al., Conf. Proc. IPAC'11, MOPS014.

[10] C.Hansen et al., Conf. Proc. IPAC'11, WEPS002.

[11] A.Chancé, et al., Conf. Proc. IPAC'11, WEPS001.

[12] M.Benedikt et al., Eur.Phys.J.A47(2011) 24. 